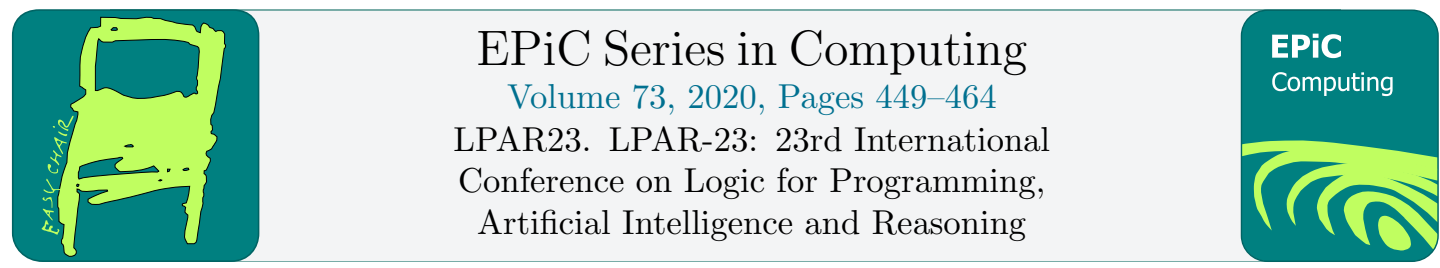

\title{
On Reasoning about Access to Knowledge
}

\author{
Yakoub Salhi \\ CRIL CNRS \& Univ. Artois, \\ F-62300 Lens, France \\ salhi@cril.fr
}

\begin{abstract}
Controlling access to knowledge plays a crucial role in many multi-agent systems. Indeed, it is related to different central aspects in interactions among agents such as privacy, security, and cooperation. In this paper, we propose a framework for dealing with access to knowledge that is based on the inference process in classical propositional logic: an agent has access to every piece of knowledge that can be derived from the available knowledge using the classical inference process. We first introduce a basic problem in which an agent has to hide pieces of knowledge, and we show that this problem can be solved through the computation of maximal consistent subsets. In the same way, we also propose a counterpart of the previous problem in which an agent has to share pieces of knowledge, and we show that this problem can be solved through the computation of minimal inconsistent subsets. Then, we propose a generalization of the previous problem where an agent has to share pieces of knowledge and hide at the same time others. In this context, we introduce several concepts that allow capturing interesting aspects. Finally, we propose a weight-based approach by associating integers with the pieces of knowledge that have to be shared or hidden.
\end{abstract}

\section{Introduction}

Recent technological advances have brought about numerous important problems related to knowledge exchange between different systems. In particular, (autonomous) agents can be led in many contexts to withhold and share knowledge to achieve specific purposes. This creates the need of processes for controlling and reasoning about knowledge transfer. Indeed, such processes can play a key role in various forms of interaction among agents. For instance, in automated negotiation [15, 20,11], the relevant and useful strategies are often based on the shared arguments and information. In addition, to preserve privacy in multi-agent systems [10, 23], autonomous agents have to hide information about their critical principals, such as preferences, beliefs and other information. Furthermore, in cooperation in multi-agent systems [8, 17], agents attempt to jointly solve tasks, and to this end their communication plays a central role. The main problem that we emphasize in this work is the difficulty of finding compromises between knowledge hiding and sharing.

We use in this work classical propositional logic for knowledge representation and reasoning. Indeed, the knowledge of an agent as well as the shared information are represented by

E. Albert and L. Kovacs (eds.), LPAR23 (EPiC Series in Computing, vol. 73), pp. 449-464 
propositional formulas. The use of a logical-based approach is mainly motivated by the fact that it offers flexible representation models [21]. It is worth noting that propositional logic has been used to model beliefs, preferences and knowledge in several domains, including argumentation [5], belief base merging [14], and belief revision [1]. In particular, the process of inference is appropriate in many situations for formally updating and deriving new representations. We consider in this paper that every agent has access to every piece of knowledge that can be derived using this process from the pieces of knowledge in her possession. That being said, it is worth mentioning that our framework can be directly adapted to other processes of inference, in particular those of formal logics based on classical reasoning such as normal modal logics [6] and description logics [3].

Our main contribution in this paper is a logic-based framework for reasoning about knowledge access. We first describe a basic problem in which an agent has to hide pieces of knowledge in interaction with another agent. We introduce in this context a concept that plays a key role in this problem called minimal hiding subset (MHS). The MHSes are subsets of pieces of knowledge that can be shared by an agent trying to hide certain knowledge so that if these pieces are hidden then the purpose of the agent is achieved. These subsets have to be minimal with respect to set-theoretic inclusion. We show that the extraction of MHSes can be carried out through the extraction of particular maximal consistent subsets. Regarding computational complexity, we show that the problem of determining if a subset of formulas is an MHS is $D^{P}$-complete. Furthermore, we propose a counterpart of the notion of MHS called minimal sharing subset (MSS), and we show that the extraction of MSSes can be solved through the computation of minimal inconsistent subsets. We also show that the problem of determining if a subset of formulas is an MSS is $D^{P}$-complete.

Subsequently, we propose a generalization by allowing reasoning about both knowledge sharing and hiding simultaneously. Intuitively, the main idea consists in providing approaches describing types of compromise between hiding enough and sharing enough. Similarly to MHSes, we introduce two new concepts called maximal SH-subset and minimal SH-subset. The former consists in a subset of the available pieces of knowledge that have no superset that allows hiding the same knowledge, while the latter has no subset that allows sharing the same knowledge. In addition, we propose particular types of maximal and minimal SH-subsets that allow capturing interesting forms of compromise between hiding and sharing. In particular, we introduce the preferred maximal and minimal SH-subsets that are defined as the SH-subsets that cannot be improved in the sense of allowing the agent to share and hide more pieces of knowledge w.r.t. her purpose. It is worth mentioning that we provide computational complexity results related to the computation of these types of SH-subsets.

Finally, we propose a weight-based approach by associating integers with the pieces of knowledge that have to be shared or hidden. This approach is based on the assumption that the agent has to adopt a strategy in selecting pieces of knowledge that maximizes the weight. In this regard, we particularly consider bi-objective optimization by taking into account the weight of sharing as well as that of hiding knowledge.

The paper is organized as follows. In Section 2, we describe some preliminary definitions and notational conventions. Section 3 introduces the concept of minimal hiding subset. Section 4 provides results on computing the minimal hiding subsets. Section 5 introduces the concept of minimal sharing subset. Section 6 gives our generalization by combining knowledge hiding and sharing. Section 7 is devoted to the description of our weight-based approach. Section 8 concludes the paper and provides some perspectives. 


\section{Preliminaries}

In this section, we describe the syntax and semantics of classical propositional logic, and some notational conventions.

We use Prop to denote the set of propositional variables. The propositional formulas of classical propositional logic $(C P L)$ are built using Prop, the constants $\top$ and $\perp$, denoting respectively true and false, and the unary logical connective $\neg$ and the usual binary connectives $\wedge, \vee$ and $\rightarrow$. The grammar is defined as follows:

$$
\phi::=p|\top| \perp|(\phi)| \phi \wedge \phi|\phi \vee \phi| \phi \rightarrow \phi \mid \neg \phi
$$

with $p \in$ Prop. The set of propositional formulas is denoted Form. We use the letters $p, q, r, s$ to denote the propositional variables, and the Greek letters $\phi, \psi$ and $\chi$ to denote the propositional formulas. Moreover, given a syntactic object $o$ involving propositional variables (a formula, a set of formulas, etc.), we use $\operatorname{Var}(o)$ to denote the set of propositional variables occurring in $o$.

Note that brackets can be omitted by assuming that $\neg$ has higher precedence than $\wedge$, and this connective has higher precedence than $\vee$ which has higher precedence than $\rightarrow$. Moreover, we write $\left(\phi_{1} \wedge \phi_{2} \wedge \phi_{3} \wedge \cdots \wedge \phi_{n}\right)$ and $\left(\phi_{1} \vee \phi_{2} \vee \phi_{3} \vee \cdots \vee \phi_{n}\right)$ instead of respectively $\left(\cdots\left(\left(\phi_{1} \wedge\right.\right.\right.$ $\left.\left.\left.\phi_{2}\right) \wedge \phi_{3}\right) \wedge \cdots \wedge \phi_{n}\right)$ and $\left(\cdots\left(\left(\phi_{1} \vee \phi_{2}\right) \vee \phi_{3}\right) \vee \cdots \vee \phi_{n}\right)$.

A Boolean interpretation $\mathcal{B}$ of a formula $\phi$ is defined as a function from a set of variable $V$, with $\operatorname{Var}(\phi) \subseteq V$, to $\{0,1\}$ ( 0 stands for false and 1 for true). It is inductively extended to the propositional formulas as usual:

$$
\begin{array}{cccc}
\mathcal{B}(\top)=1 & \mathcal{B}(\perp)=0 & \mathcal{B}(\neg \phi)=1-\mathcal{B}(\phi) & \mathcal{B}(\phi \wedge \psi)=\min (\mathcal{B}(\phi), \mathcal{B}(\psi)) \\
\mathcal{B}(\phi \vee \psi)=\max (\mathcal{B}(\phi), \mathcal{B}(\psi)) & \mathcal{B}(\phi \rightarrow \psi)=\max (1-\mathcal{B}(\phi), \mathcal{B}(\psi))
\end{array}
$$

A formula $\phi$ is consistent if there exists a Boolean interpretation $\mathcal{B}$ of $\phi$ such that $\mathcal{B}(\phi)=1$, and $\mathcal{B}$ is called a model in this case. Given a set of variables $S$ such that $\operatorname{Var}(\phi) \subseteq S$, we use $\operatorname{Mod}(\phi, S)$ to denote the set of all models of $\phi$ defined over $S$. The formula $\phi$ is said to be valid or a theorem, if every Boolean interpretation of $\phi$ is one of its model.

Given a finite set of formulas $\Gamma$, a formula $\phi$ is said to be a logical consequence of $\Gamma$, written $\Gamma \vdash \phi$, if $\Lambda \Gamma \rightarrow \phi$ is a valid formula, where $\bigwedge \Gamma$ stands for the conjunction of the formulas occurring in $\Gamma$ with $\bigwedge \emptyset=\top$. Equivalently, $\Gamma \vdash \phi$ holds if every model in $\operatorname{Mod}(\bigwedge \Gamma, \operatorname{Var}(\Gamma \cup\{\phi\}))$ is also a model of $\phi$. In the case where $\Gamma$ contains a single formula $\psi$, we write $\psi \vdash \phi$. In addition, if both $\phi \vdash \psi$ and $\psi \vdash \phi$ hold, we write $\phi \equiv \psi$. In particular, the connective $\leftrightarrow$ is defined as follows: $\phi \leftrightarrow \psi \equiv(\phi \rightarrow \psi) \wedge(\psi \rightarrow \phi)$.

\section{Minimal Hiding Subset}

In this section, we introduce a basic problem in which an agent that is in interaction with another agent has to hide pieces of knowledge. We consider that access to knowledge is based on the inference process in classical propositional logic. More precisely, we consider that every agent has access to every piece of knowledge that can be derived from the available knowledge using the classical inference process. Figure 1 describes the intuition behind our framework. In this figure, the agent on the left (agent $\left.{ }_{1}\right)$ wants to hide both $B$ and $C$, and knowing that the agent on the right $\left(\right.$ agent $_{2}$ ) can deduce $B$ and $C$ from $A$, agent $_{1}$ avoids sharing $A$.

In the following definition, we introduce a concept that is used to describe situations where an agent, in an interaction with another agent, has to hide pieces of knowledge. 


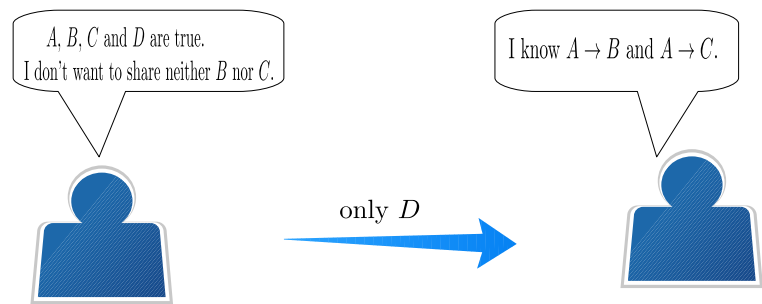

Figure 1: An illustration for knowledge hiding.

Definition 1 (HI-frame). A HI-frame is a tuple $F=(K, \phi, H)$ consisting of a propositional formula $\phi$ and two finite sets of propositional formulas $K$ and $H$ where $K \cup\{\phi\} \nvdash \perp$.

The set of formulas $K$ is used to represent the pieces of knowledge that can be conveyed by the agent that is trying to hide certain knowledge, called the principal agent, the formula $\phi$ corresponds to the knowledge of the other agent involved in the interaction, called the secondary agent, and $H$ is the knowledge that the principle agent wants to hide.

Let us now introduce a key notion that is used to determine the pieces of knowledge that have to be hidden.

Definition 2 (Minimal Hiding Subset). A Minimal Hiding Subset (MHS) of a HI-frame F= $(K, \phi, H)$ is a subset of propositional formulas $E \subseteq K$ that satisfies the two following properties:

(i) for all $\psi \in H,(K \backslash E) \cup\{\phi\} \nvdash \psi$; and

(ii) for all $E^{\prime} \subset E$, there exists $\psi \in H$ s.t. $\left(K \backslash E^{\prime}\right) \cup\{\phi\} \vdash \psi$.

In other words, an MHS is a subset of the pieces of knowledge that can be conveyed by the principal agent (i.e. a subset of $K$ ) so that if all the other formulas are shared with the secondary agent (i.e. those belonging to $K \backslash E$ ), then the purpose of the principal agent is achieved: the formulas that have to be hidden to this agent remain in this situation (i.e. $(K \backslash E) \cup\{\phi\} \nvdash \psi$ holds for every $\psi \in H$ ); moreover, an MHS must be minimal w.r.t. set-theoretic inclusion ( $(i)$ ensures the objective of hiding while (ii) ensures the minimality of $E$ )

Example 1. The situation described in this example involves a person and a company. Consider the following statements about the person:

- she supports at least one of the public policies $p_{1}$ and $p_{2}\left(p_{1} \vee p_{2}\right)$;

- if she supports the public policy $p_{1}$, she also supports the bill $b_{1}\left(p_{1} \rightarrow b_{1}\right)$; and

- if she supports the public policy $p_{2}$, she also supports the bill $b_{2}\left(p_{2} \rightarrow b_{2}\right)$.

The company holds the following piece of knowledge:

- if a person supports at least one of the bills $b_{1}, b_{2}$, and $b_{3}$, then she will vote for the candidate $c\left(\left(b_{1} \vee b_{2} \vee b_{3}\right) \rightarrow c\right)$.

Clearly, from the previous pieces of knowledge, we know that the considered person will vote for the candidate $c$. In this context, the aim is to hide this information, which can be described by the HI-frame $F=\left(\left\{p_{1} \vee p_{2}, p_{1} \rightarrow b_{1}, p_{2} \rightarrow b_{2}\right\},\left(b_{1} \vee b_{2} \vee b_{3}\right) \rightarrow c,\{c\}\right)$. In this regard, one can easily see that the HI-frame $F$ admits only three minimal hiding subsets, which are $E_{1}=\left\{p_{1} \vee p_{2}\right\}, E_{2}=\left\{p_{1} \rightarrow b_{1}\right\}$ and $E_{3}=\left\{p_{2} \rightarrow b_{2}\right\}$. Indeed, hiding any of the three previous sets allows us to avoid sharing $b_{1} \vee b_{2}$, and consequently the company cannot derive $c$. 


\section{On Computing Minimal Hiding Subsets}

We study in this section aspects related to the problem of computing the minimal hiding subsets of a given HI-frame. In particular, we show that this problem can be solved through the computation of maximal consistent subsets. More precisely, we associate with each HI-frame a set of formulas so that the maximal consistent subsets containing a specific formula correspond to all the minimal hiding subsets of the considered HI-frame. Regarding computational complexity, we show that the problem of determining if a set of formulas is an MHS of a given HI-frame is $D^{P}$-complete.

Definition 3 (Maximal Consistent Subset). Let $K$ be a finite set of propositional formulas and $M \subseteq K$. The subset $M$ is said to be maximal consistent subset (MCS) of $K$ if the following two properties hold:

- $M \nvdash \perp$, and

- for all $\phi \in K \backslash M, M \cup\{\phi\} \vdash \perp$.

It is worth noting that there are several methods for computing MCSes (e.g. for a recent work see [12]). This comes from the fact that they are particularly useful in knowledge representation and reasoning. Indeed, various approaches for reasoning under inconsistency are based on the use of MCSes (e.g. $[4,5,2])$.

Now, we introduce our encoding for computing the MHSes of a given HI-frame. Let $F=(K, \phi, H)$ be a HI-frame. In order to define our encoding for $F$, we associate with each propositional variable $p$ occurring in $F$ a set of $m$ distinct fresh propositional variables denoted $p^{1}, \ldots, p^{m}$ where $m=|H|$. In this context, we use $f$ to refer to an arbitrary bijective function from $H$ to $\{1, \ldots, m\}$. Then, given a propositional formula $\psi$ occurring in $F$, we use $(\psi)^{i}$, with $i \in 1 . . m$, to denote the formula obtained from $\psi$ by replacing each variable $p$ in $\psi$ with $p^{i}$ (e.g. $\left.((p \wedge q) \rightarrow r)^{i}=\left(p^{i} \wedge q^{i}\right) \rightarrow r^{i}\right)$. We use $\mathcal{E}_{M H S}(F)$ to denote the following set of $|K|+1$ formulas:

$$
\left\{\bigwedge_{i=1}^{m}(\psi)^{i}: \psi \in K\right\} \cup\left\{\bigwedge_{\chi \in H}(\phi \wedge \neg \chi)^{f(\chi)}\right\}
$$

Intuitively, $\bigwedge_{i=1}^{m}(\psi)^{i}$ is used to express that $\psi$ is consistent with $\phi$ and the negation of every formula occurring in $H$. In this regard, the truth of $\bigwedge_{\chi \in H}(\phi \wedge \neg \chi)^{f(\chi)}$ allows avoiding the derivation of any element of $H$.

The following proposition shows how the MHSes can be directly obtained from the MCSes of $\mathcal{E}_{M H S}(F)$ that contain the formula $\bigwedge_{\chi \in H}(\phi \wedge \neg \chi)^{f(\chi)}$.

Proposition 1 (Soundness). Let $F=(K, \phi, H)$ be a HI-frame and $E \subseteq K$. Then, $E$ is an $M H S$ of $F$ iff $M=\left\{\bigwedge_{i=1}^{|H|}(\psi)^{i}: \psi \in K \backslash E\right\} \cup\left\{\bigwedge_{\chi \in H}(\phi \wedge \neg \chi)^{f(\chi)}\right\}$ is an MCS of $\mathcal{E}_{M H S}(F)$.

Proof.

Part $\Rightarrow$. Using the fact that $E$ is an MHS of $F$, we obtain $(K \backslash E) \cup\{\phi\} \cup\{\neg \chi\} \nvdash \perp$ for every $\chi \in H$. Thus, $\left\{(\psi)^{f(\chi)}: \psi \in(K \backslash E) \cup\{\phi\} \cup\{\neg \chi\}\right\} \nvdash \perp$ holds for every $\chi \in H$. Therefore, knowing that $(\psi)^{i}$ and $(\psi)^{j}$ do not share any variable for every formula $\psi$ occurring in $F$ and for every two distinct integers $i, j \in 1 . .|H|$, we obtain $M \nvdash \perp$. Assume now that $M$ is not an MCS. Then, there exists $\psi \in E$ s.t. $M \cup\left\{\bigwedge_{i=1}^{|H|}(\psi)^{i}\right\} \nvdash \perp$ holds. As a consequence, we have $(K \backslash E) \cup\{\psi\} \cup\{\phi\} \cup\{\neg \chi\} \nvdash \perp$ for every $\chi \in H$; thereby, we obtain a contradiction since $E$ is an MHS of $F$.

Part $\Leftarrow$. Using the property $M \nvdash \perp$, we have $(K \backslash E) \cup\{\phi\} \cup\{\neg \chi\} \nvdash \perp$ for every $\chi \in H$, 
since $(\psi)^{i}$ and $(\psi)^{j}$ do not share any variable for every formula $\psi$ occurring in $F$ and for every two distinct integers $i, j \in 1 . .|H|$. Then, we obtain $(K \backslash E) \cup\{\phi\} \nvdash \chi$ for every $\chi \in H$. Moreover, using the fact that $M$ is an MCS, $M \cup\left\{\bigwedge_{i=1}^{|H|}(\psi)^{i}\right\} \vdash \perp$ for every $\psi \in E$. Thus, for all $\psi \in E$ there exists $\chi \in H$ s.t. $(K \backslash E) \cup\{\phi\} \cup\{\psi\} \cup\{\neg \chi\} \vdash \perp$ holds, which implies $(K \backslash E) \cup\{\phi\} \cup\{\psi\} \vdash \chi$. Therefore, $E$ is an MHS of $F$.

Let us now show the problem of determining if a subset of formulas is an MHS is $D^{P}$ complete. The complexity class $D^{P}$ is defined as follows: a language $L$ is in $D^{P}$ if and only if there are two languages $L_{1} \in \mathrm{NP}$ and $L_{2} \in \mathrm{CO}-\mathrm{NP}$ such that $L=L_{1} \cap L_{2}[19,18]$. This class is also known as the second level of the Boolean hierarchy, BH2. It is worth mentioning that the previous property does not mean that $D^{P}$ is equal to NP $\cap$ CO-NP. One of the well-known $D^{P}$-complete problems is SAT-UNSAT: given an ordered pair of propositional formulas $(\phi, \psi)$, determine if $\phi$ is satisfiable and $\psi$ is unsatisfiable. In the proof of the following theorem, we use the $D^{P}$-complete problem of determining if a set of formulas is a maximal consistent subset of a given set of propositional formulas [7].

Theorem 1. The problem of determining if a set of formulas is an MHS of a given HI-frame is $D^{P}$-complete.

Proof. The fact that the considered decision problem is in $D^{P}$ is a direct consequence of our encoding $\mathcal{E}_{M H S}(F)$. Indeed, the problem of determining if a set of formulas is an MCS of a given set of formulas is $D^{P}$-complete (Pb-MCS). Let us now show $D^{P}$-hardness. To this end, we provide a reduction of the problem Pb-MCS using an approach similar to the encoding $\mathcal{E}_{M H S}(F)$. Let $K$ be a finite set of propositional formulas. We consider w.l.o.g. that all the formulas in $K$ are consistent. To define our reduction, we associate $n$ distinct fresh variables $p_{1}, \ldots, p_{n}$ with each propositional variable $p$ occurring in $K$ where $n=|K|$. Moreover, given a formula $\psi \in K$, we use $(\psi)^{i}$ to denote the formula obtained from $\psi$ by replacing every variable $p$ occurring in $\psi$ with the fresh variable $p_{i}$. In addition, we use $f$ to refer to an arbitrary bijective function from $K$ to $\{1, \ldots, n\}$. Consider now the following HI-frame

$$
\begin{aligned}
& \overbrace{n-1}^{\phi} \\
& F=\left(\left\{(\psi)^{f(\psi)}: \psi \in K\right\}, q \leftrightarrow\left(\bigwedge_{p \in \operatorname{Var}(K)} \bigwedge_{i=1}\left(p_{i} \leftrightarrow p_{i+1}\right)\right),\{\neg q\}\right)
\end{aligned}
$$

Given a subset $M \subseteq K$. Clearly, $M$ is an MCS of $K$ if and only if $\left\{(\psi)^{f(\psi)}: \psi \in K \backslash M\right\}$ is an MHS of $F$. In particular, the fact that $M$ is consistent comes from the fact $\left\{(\psi)^{f(\psi)}\right.$ : $\psi \in M\} \cup\{\phi\} \cup\{q\}$ is consistent. Furthermore, $M$ is maximal w.r.t. set inclusion because $\left\{(\psi)^{f(\psi)}: \psi \in K \backslash M\right\}$ is minimal w.r.t. set inclusion.

\section{A Counterpart: Minimal Sharing Subset}

In this section, we introduce in a simple way a counterpart of the notion of minimal hiding subset that is related to knowledge sharing. The aim in this variant is to reduce the number of conveyed formulas to allow the principal agent to achieve her purpose, which consists in sharing certain pieces of knowledge.

Let us first present the notion of SI-frame, which is also defined as a tuple $F=(K, \phi, S)$ that satisfies the same properties as those in the case of HI-frame, in particular the property $K \cup\{\phi\} \nvdash \perp$. In the same way as in a HI-frame, the set $K$ represents the pieces of knowledge 
that can be conveyed and $\phi$ the knowledge of the secondary agent; however, the set $S$ represents the pieces of knowledge that the principal agent wants to share.

Definition 4 (Minimal Sharing Subset). A Minimal Sharing Subset (MSS) of a given SIframe $F=(K, \phi, S)$ is a subset of propositional formulas $E \subseteq K$ that satisfies the two following properties:

(i) for all $\psi \in S, E \cup\{\phi\} \vdash \psi$; and

(ii) for all $E^{\prime} \subset E$, there exists $\psi \in S$ s.t. $E^{\prime} \cup\{\phi\} \nvdash \psi$.

The property $(i)$ allows guaranteeing that the purpose of the principal agent is achieved, and the property $(i i)$ allows obtaining that an MSS is minimal w.r.t. set inclusion.

For instance, consider the following SI-frame: $F=(\{p, q, r\},(p \rightarrow q) \wedge(q \leftrightarrow r),\{p, q, r\})$. It admits a unique MSS, which is $\{p\}$. Indeed, sharing $p$ allows the principal agent to share all the desired pieces of knowledge: the piece of knowledge $q$ is obtained from $p \rightarrow q$ and $r$ from $q \leftrightarrow r$.

In order to compute the MSSes of a given SI-frame, we propose an approach based on computing minimal inconsistent subsets of an associated set of formulas.

Definition 5 (Minimal Inconsistent Subset). Let $K$ be a finite set of propositional formulas and $M \subseteq K$. The subset $M$ is said to be minimal inconsistent subset (MIS) of $K$ if the following properties hold:

- $M \vdash \perp$, and

- for all $\phi \in M, M \backslash\{\phi\} \nvdash \perp$.

Our encoding for computing the MSSes of a given SI-frame is simpler than that used for computing the MHSes of a given HI-frame, described in Section 4. Let $F=(K, \phi, S)$ be an SI-frame. We use $\mathcal{E}_{M S S}(F)$ to denote the following set of formulas:

$$
K \cup\left\{\phi \wedge \bigvee_{\chi \in S} \neg \chi\right\}
$$

The soundness of our approach is a direct consequence of the fact that we have $E \cup\{\phi \wedge$ $\left.\bigvee_{\chi \in S} \neg \chi\right\} \vdash \perp$ if and only if we have $E \cup\{\phi\} \vdash \bigwedge_{\chi \in S} \chi$.

Proposition 2 (Soundness). Let $F=(K, \phi, S)$ be an SI-frame and $E \subseteq K$. Then, $E$ is an $M S S$ of $F$ iff $M=E \cup\left\{\phi \wedge \bigvee_{\chi \in S} \neg \chi\right\}$ is a MIS of $\mathcal{E}_{M S S}(F)$.

In the same way as for the computation of MCSes, there are many methods in the literature that are dedicated to the computation of MISes (e.g. see [16]). As a side note, the MISes are also widely used for reasoning under inconsistency (e.g. in building arguments [5] and measuring inconsistency [13]).

The following theorem shows that the problem of determining whether a set of formulas is an MSS belongs to the same computational complexity class as that of determining whether a set of formulas is an MHS.

Theorem 2. The problem of determining if a set of formulas is an MSS of a given SI-frame is $D^{P}$-complete. 
Proof. Proposition 2 shows that the considered decision problem belongs to $D^{P}$, since determining if a subset of formulas is a minimal consistent subset is a $D^{P}$-complete problem (MIS-Pb). In order to show $D^{P}$-hardness, we provide an encoding of MIS-Pb. Let $M=\left\{\phi_{1}, \ldots, \phi_{k}\right\}$ be a finite set of formulas. We associate with each element $\phi_{i}$ of $M$ a distinct fresh propositional variable denoted $p_{i}$. Then our encoding in the case of $M$ is defined by the following SI-frame:

$$
F_{M}=\left(\left\{p_{1}, \ldots, p_{k}\right\}, \top,\left\{\neg\left(p_{1} \leftrightarrow \phi_{1}\right) \vee \cdots \vee \neg\left(p_{k} \leftrightarrow \phi_{k}\right)\right\}\right)
$$

By mainly using the fact that $p_{1}, \ldots, p_{k}$ are distinct fresh propositional variables, $E=$ $\left\{p_{1}, \ldots, p_{k}\right\}$ is an MSS of $F_{M}$ if and only if $E \cup\left\{\left(p_{1} \leftrightarrow \phi_{1}\right) \wedge \cdots \wedge\left(p_{k} \leftrightarrow \phi_{k}\right)\right\} \vdash \perp$, which means $M \vdash \perp$, and for every $E^{\prime} \subset E, E^{\prime} \cup\left\{\left(p_{1} \leftrightarrow \phi_{1}\right) \wedge \cdots \wedge\left(p_{k} \leftrightarrow \phi_{k}\right)\right\} \nvdash \perp$, since otherwise $E$ is not minimal w.r.t. set inclusion. As a consequence, $M$ is a minimal inconsistent subset.

\section{Combining Knowledge Sharing and Hiding}

We intend in this section to propose a framework for reasoning about both knowledge sharing and hiding simultaneously. Indeed, we introduce different generalizations of the two problems of knowledge hiding and sharing described previously. The main idea consists in providing approaches describing types of compromises for solving possible conflicts between knowledge sharing and hiding. Figure 2 describes a situation showing such conflicts. Indeed, if the principal agent conveys the two pieces of knowledge $p$ and $q$, then the secondary agent can derive a piece of knowledge that she wants to hide, namely $r$. Thus, in order to hide $r$, the principal agent has to hide also $p$ or $q$.

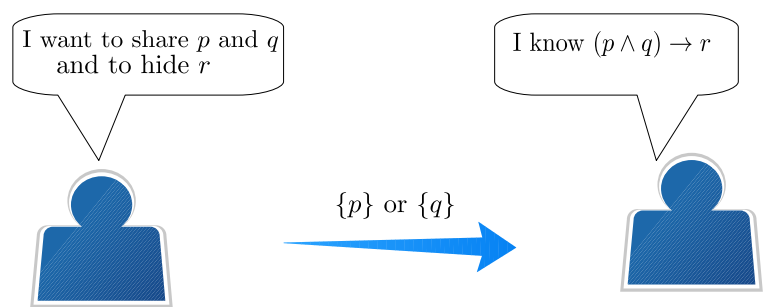

Figure 2: An illustration for knowledge hiding and sharing.

The concept introduced in the following definition can be seen as a generalization of both HI-frames and SI-frames.

Definition 6 (SHI-Frame). A SHI-frame is a tuple $F=(K, \phi, H, S)$ consisting of a propositional formula $\phi$ and three finite sets of propositional formulas $K, H$ and $S$ where $K \cup\{\phi\} \nvdash \perp$.

Similarly to the HI-frames and the SI-frames, the set $K$ represents the pieces of knowledge that can be conveyed and $\phi$ the knowledge of the secondary agent. The sets $H$ and $S$ represent respectively the pieces of knowledge that the principal agent wants to hide and those that she wants to share.

In the following two definitions, we introduce two notions that are similar to those of minimal hiding subset and minimal sharing subset. 
Definition 7 (Maximal SH-Subset). A maximal SH-subset (in short MaxSHS) of a SHI-frame $F=(K, \phi, H, S)$ is a subset of propositional formulas $E \subseteq K$ s.t. for all $E^{\prime} \supset E,\{\psi \in H$ : $\left.E^{\prime} \cup\{\phi\} \nvdash \psi\right\} \subset\{\psi \in H: E \cup\{\phi\} \nvdash \psi\}$.

Definition 8 (Minimal SH-Subset). A minimal SH-subset (in short MinSHS) of a SHI-frame $F=(K, \phi, H, S)$ is a subset of propositional formulas $E \subseteq K$ s.t. for all $E^{\prime} \subset E,\{\psi \in S$ : $\left.E^{\prime} \cup\{\phi\} \vdash \psi\right\} \subset\{\psi \in S: E \cup\{\phi\} \vdash \psi\}$.

Alternatively stated, a maximal SH-subset is subset of the available pieces of knowledge that has no superset that allows hiding the same formulas from the secondary agent; while a minimal $\mathrm{SH}$-subset has no subset that allows sharing the same formulas. We particularly consider these two notions to take into account situations where the principal agent has to reduce the set of conveyed formulas as well as those where she has to enlarge this set as far as possible w.r.t. the considered purpose. It is easy to see that a MaxSHS (resp. MinSHS) allows the principal agent to share (resp. hide) the same formulas as their supersets (resp. subsets). Roughly speaking, these notions allow us to consider the cases where the principal agent has to make minimal and maximal changes to the available pieces of knowledge for approaching her purpose about combining knowledge hiding and sharing.

Let us now introduce interesting particular sorts of maximal and minimal SH-subsets. The main objective is to avoid the SH-subsets that can be improved with respect to the purpose of the principal agent.

Definition 9 (Admissible Maximal SH-Subset). An admissible MaxSHS of a SHI-frame $F=$ $(K, \phi, H, S)$ is a MaxSHS $E$ of $F$ that satisfies the following property:

$\forall E^{\prime} \subset E$ with $\left\{\psi \in S: E^{\prime} \cup\{\phi\} \vdash \psi\right\}=\{\psi \in S: E \cup\{\phi\} \vdash \psi\},\left\{\psi \in H: E^{\prime} \cup\{\phi\} \nvdash \psi\right\}=$ $\{\psi \in H: E \cup\{\phi\} \nvdash \psi\}$.

Definition 10 (Admissible Minimal SH-Subset). An admissible MinSHS of a SHI-frame $F=$ $(K, \phi, H, S)$ is a MinSHS $E$ of $F$ that satisfies the following property:

$\forall E^{\prime} \supset E$ with $\left\{\psi \in H: E^{\prime} \cup\{\phi\} \nvdash \psi\right\}=\{\psi \in H: E \cup\{\phi\} \nvdash \psi\},\left\{\psi \in S: E^{\prime} \cup\{\phi\} \vdash \psi\right\}=$ $\{\psi \in S: E \cup\{\phi\} \vdash \psi\}$.

A maximal (resp. minimal) SH-subset is admissible if it cannot be improved, in the sense of allowing the principal agent to share and hide more pieces of knowledge, by considering one of its subsets (resp. supersets). The admissible SH-subsets can be used when the principal agent seeks the best solutions that involve specific formulas.

In the following definition, we use a similar idea to the definition of admissible SH-subsets but we consider any subset of the available pieces of knowledge instead of the subsets and the supersets of the SH-subsets. For instance, a maximal SH-subset is preferred if there is no subset of the available pieces of knowledge that allows sharing and hiding more.

Definition 11 (Preferred SH-Subset). A preferred MaxSHS (resp. preferred MinSHS) of a SHI-frame $F=(K, \phi, H, S)$ is a subset of propositional formulas $E \subseteq K$ s.t.

(i) E is a MinSHS (resp. MaxSHS) of F; and

(ii) for all $E^{\prime} \subseteq K$, we have:

- if $\{\psi \in H: E \cup\{\phi\} \nvdash \psi\} \subset\left\{\psi \in H: E^{\prime} \cup\{\phi\} \nvdash \psi\right\}$ then $\{\psi \in S: E \cup\{\phi\} \vdash \psi\} \not \subset$ $\left\{\psi \in S: E^{\prime} \cup\{\phi\} \vdash \psi\right\}$, and 
- if $\{\psi \in S: E \cup\{\phi\} \vdash \psi\} \subset\left\{\psi \in S: E^{\prime} \cup\{\phi\} \vdash \psi\right\}$ then $\{\psi \in H: E \cup\{\phi\} \nvdash \psi\} \not \subset$ $\left\{\psi \in H: E^{\prime} \cup\{\phi\} \nvdash \psi\right\}$.

The following proposition is a direct consequence of the definitions of admissible and preferred MaxSHSes and MinSHSes.

Proposition 3. If $E$ is a preferred MaxSHS (resp. MinSHS) of a SHI-frame $F$ then it is an admissible MaxSHS (resp. MinSHS) of F.

Example 2. Consider the following SHI-frame:

$$
F=\left(\left\{p, p^{\prime}, q, r, s, t\right\},((p \wedge q) \rightarrow r) \wedge\left(p^{\prime} \rightarrow(p \wedge u)\right),\{r, t\},\{p, q, u\}\right)
$$

Thus, the aim in $F$ is to hide the formulas $r$ and $t$, and to share $p, q$ and $u$. For instance, the sets $\left\{p, p^{\prime}, q, r, s, t\right\}$ and $\emptyset$ are respectively a MaxSHS and a MinSHS of F. In addition, the previous two sets are both not admissible. Indeed, $\left\{p, p^{\prime}, q, r, s, t\right\}$ is not admissible since $\left\{p, p^{\prime}, q, r, s\right\}$ is a MaxSHS that hides more pieces of knowledge and shares the same desired knowledge; and $\emptyset$ is not admissible since $\{p\}$ is a MinSHS that allows hiding $r$ and $t$ and sharing $p$. The admissible MinSHSes of $F$ are only $\{p\},\left\{p^{\prime}\right\}$ and $\{q\}$. Furthermore, the admissible MaxSHSes of $F$ are $\left\{p, p^{\prime}, q, r, s\right\},\left\{p, p^{\prime}, s\right\}$ and $\{q, s\}$. In addition, the set $\{p\}$ is not a preferred MinSHS since $\left\{p^{\prime}\right\}$ allows hiding the same knowledge with sharing more desired pieces of knowledge. The preferred MinSHSes (resp. MaxSHSes) of $F$ are $\left\{p^{\prime}\right\}$ and $\{q\}$ (resp. $\left\{p, p^{\prime}, q, r, s\right\},\left\{p, p^{\prime}, s\right\}$ and $\{q, s\}$ ).

It is worth mentioning that, for evey SHI-frame $F=(K, \phi, H, S)$, the set $K$ is a MaxSHS and $\emptyset$ is a MinSHS of $F$, but not necessarily admissible (see Example 2).

In the following definition, we describe decision problems related to the computation of admissible and preferred MaxSHSes and MinSHSes. These problems are used for presenting some important computational complexity aspects.

Definition 12. The decision problems AdMaxSHS, AdMinSHS, PrMaxSHS and PrMinSHS are defined as follows:

- AdMaxSHS: determining if a MaxSHS is admissible;

- AdMinSHS: determining if a MinSHSs is admissible;

- PrMaxSHS: determining if a MaxSHS is preferred;

- PrMinSHS: determining if a MinSHS is preferred.

The polynomial hierarchy is a hierarchy of computational complexity classes that generalize the well-known classes $P, \mathrm{NP}$ and CO-NP [22]. In particular, the class $\Sigma_{2}^{P}$ is that of the problems that can be decided by an NP-algorithm using an NP-oracle. Similarly, the class $\Pi_{2}^{P}$ is that of the problems that can be decided by a CO-NP-algorithm using an NP-oracle, which means that $\Pi_{2}^{P}=c o-\Sigma_{2}^{P}$. One of the well-known $\Sigma_{2}^{P}$-complete problem is a restriction of the Quantified SAT problem to particular quantified Boolean formulas (QBF). More precisely, the problem of deciding whether a given $\mathrm{QBF}$ of the form $\exists p_{1} \cdots \exists p_{k} \forall q_{1} \cdots \forall q_{l} \Omega$ (with $\Omega$ a propositional formula) is true $\left(Q S A T_{2}\right)$ is $\Sigma_{2}^{P}$-complete. It is worth mentioning that the more general problem of deciding whether a $\mathrm{QBF}$ formula is true is $P S P A C E$-compelete.

In the following theorem, we show that the problems described in Definition 12 are all $\Pi_{2}^{P}$-complete. 
Theorem 3. The problems AdMaxSHS, AdMinSHS, PrMaxSHS and PrMinSHS are $\Pi_{2}^{P}$ complete.

Proof.

Part $\in \Pi_{2}^{P}$. In order to show that the considered problems belong to $\Pi_{2}^{P}$, we only need to show that their corresponding complement problems can be solved by an NP-algorithm using an NP-oracle. For instance, to determine that a MaxSHS $E$ of $F=(K, \phi, H, S)$ is not admissible (the complement problem of AdMaxSHS), we can first guess a subset $E^{\prime} \subset E$, and then, show using an NP-oracle that:

- $\left\{\psi \in S: E^{\prime} \cup\{\phi\} \vdash \psi\right\}=\{\psi \in S: E \cup\{\phi\} \vdash \psi\}$, and

- $\left\{\psi \in H: E^{\prime} \cup\{\phi\} \nvdash \psi\right\} \supset\{\psi \in H: E \cup\{\phi\} \nvdash \psi\}$.

Similarly, to show that $E$ is not preferred (the complement of PrMaxSHS), we can first guess $E^{\prime} \subseteq K$ and then show using NP-oracle calls that:

- $\{\psi \in H: E \cup\{\phi\} \nvdash \psi\} \subset\left\{\psi \in H: E^{\prime} \cup\{\phi\} \nvdash \psi\right\}$ and $\{\psi \in S: E \cup\{\phi\} \vdash \psi\} \subseteq\{\psi \in S$ : $\left.E^{\prime} \cup\{\phi\} \vdash \psi\right\}$; or

- $\{\psi \in S: E \cup\{\phi\} \vdash \psi\} \subset\left\{\psi \in S: E^{\prime} \cup\{\phi\} \vdash \psi\right\}$ and $\{\psi \in H: E \cup\{\phi\} \nvdash \psi\} \subseteq\{\psi \in H$ : $\left.E^{\prime} \cup\{\phi\} \nvdash \psi\right\}$.

Similar algorithms can be used in the case of the other two problems.

Part $\Pi_{2}^{P}$-hardness. This part can be obtained by using a reduction from the complement problem of $Q S A T_{2}$. In this proof, we only consider the case of AdMaxSHS, the other being similar. Let $I=\exists p_{1} \cdots \exists p_{k} \forall q_{1} \cdots \forall q_{l} \Omega$ be a QBF instance. To define our reduction, we associate to each propositional variable $p$ occurring in $V=\left\{p_{1}, \ldots, p_{k}\right\}$ two fresh variables $r_{p}$ and $r_{\neg p}$. Then, we define the components of the SHI-frame $F_{I}=(K, \phi, H, S)$ as follows:

- $K=\left\{r_{p}, r_{\neg p}: p \in V\right\}$

- $\phi=\top$

- $H=\left\{\psi=\bigvee_{p \in V}\left(r_{p} \wedge r_{\neg p}\right)\right\}$

- $S=\left\{\psi^{\prime}=\left(\left(\bigwedge_{p \in V}\left(r_{p} \rightarrow p\right) \wedge\left(r_{\neg p} \rightarrow \neg p\right)\right) \rightarrow \Omega\right) \wedge\left(\bigwedge_{p \in V}\left(r_{p} \vee r_{\neg} p\right)\right)\right\}$

Clearly, the set $K$ is a MaxSHS. Moreover, $K \vdash \psi$ and $K \vdash \psi^{\prime}$ hold. In addition, $K$ is admissible if and only if there is no subset $K^{\prime}$ of $K$ such that $K^{\prime} \nvdash \psi$ and $K^{\prime} \vdash \psi^{\prime}$. In this context, one can see that such a subset exists if and only if there is an assignment $\mathcal{A}$ for the variables in $V$ that allows satisfying $\forall q_{1} \cdots \forall q_{l} \Omega_{\downarrow \mathcal{A}}$ where $\Omega_{\downarrow \mathcal{A}}$ corresponds to any formula equivalent to $\Omega$ after removing the variables in $V$ according to the assignment $\mathcal{A}$. Indeed, a subset $K^{\prime}$ of $K$ satisfying $K^{\prime} \nvdash \psi$ and $K^{\prime} \vdash \psi^{\prime}$ can be defined from $\mathcal{A}$ by $K^{\prime}=\left\{r_{p}: p \in V\right.$ and $\left.\mathcal{A}(p)=1\right\} \cup\left\{r_{\neg p}: p \in\right.$ $V$ and $\mathcal{A}(p)=0\}$.

\section{A Weight-based Approach}

We introduce in this section a simple weight-based approach, which is obtained by taking into account the assumption that the principal agent has to adopt a plan in selecting pieces of knowledge that maximizes the weight. The main idea consists in association integers with the formulas that have to be hidden as well as those that have to be shared. 
Definition 13 (Weighted SHI-Frame). A weighted SHI-frame is a triple $(F, f, g)$ consisting of a SHI-frame $F=(K, \phi, H, S)$, a function $f$ that associates each element of $H$ with a strictly positive integer, and a function $g$ that associates each element of $S$ with a strictly positive integer.

The following definition introduces the solutions (subsets of the available pieces of knowledge) that allow maximizing the weight with regard to the formulas that are hidden as well those that are shared.

Definition 14 (Optimal Solution). Given a weighted SHI-frame $I=(F, f, g)$ with $F=$ $(K, \phi, H, S)$, a subset $K^{\prime} \subseteq K$ is said to be an optimal solution of $I$ if, for all $K^{\prime \prime} \subseteq K$, $\sum_{\psi \in H^{\prime \prime}} f(\psi)+\sum_{\psi \in S^{\prime \prime}} g(\psi) \geqslant \sum_{\psi \in H^{\prime}} f(\psi)+\sum_{\psi \in S^{\prime}} g(\psi)$ with $H^{\prime}=\left\{\psi \in H: K^{\prime} \cup\{\phi\} \nvdash \psi\right\}$, $H^{\prime \prime}=\left\{\psi \in H: K^{\prime \prime} \cup\{\phi\} \nvdash \psi\right\}, S^{\prime}=\left\{\psi \in S: K^{\prime} \cup\{\phi\} \vdash \psi\right\}$, and $S^{\prime \prime}=\left\{\psi \in S: K^{\prime \prime} \cup\{\phi\} \vdash \psi\right\}$. We use $W\left(K^{\prime}, I\right)$ to denote the value $\sum_{\psi \in H^{\prime}} f(\psi)+\sum_{\psi \in S^{\prime}} g(\psi)$.

Figure 3 provides a description of a simple application of our weight-based approach in a context involving a buyer and sellers. In this illustration, the buyer (the principal agent) seeks to find the best sellers (secondary agents). The pieces of information shared between the buyer and every seller allow determining the quality of transactions. In this context, the optimum weight allows the buyer to identify the best sellers. The same idea can be applied in cooperation in multi-agent systems: selecting the best partners to jointly solve a given task.

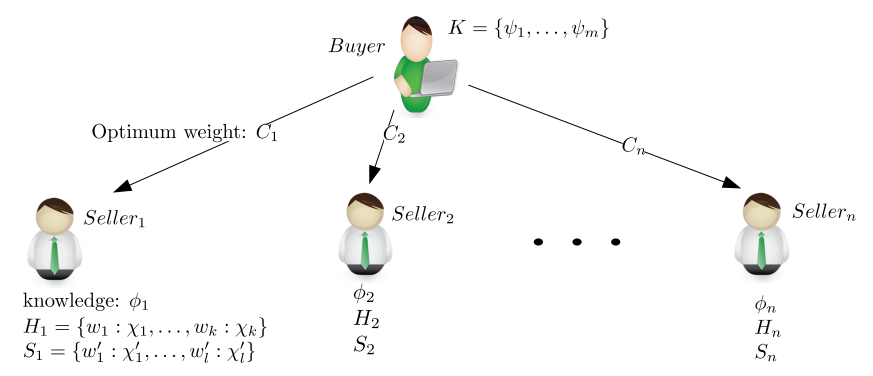

Figure 3: An illustration for a buyer seeking to find the best sellers.

The following proposition shows that optimal preferred SH-Subsets can be obtained from the optimal solutions.

Proposition 4. Given a weighted SHI-frame $I=(F, f, g)$ with $F=(K, \phi, H, S)$ and $K^{\prime} \subseteq K$, if $K^{\prime}$ is an optimal solution of $I$, then we have the two following properties:

- for all $E \subseteq K^{\prime}$, if $E$ is MinSHS s.t. $\left\{\psi \in S: K^{\prime} \cup\{\phi\} \vdash \psi\right\}=\{\psi \in S: E \cup\{\phi\} \vdash \psi\}$ then $E$ is preferred and optimal;

- for all $E \subseteq K$ with $K^{\prime} \subseteq E$, if $E$ is MaxSHS s.t. $\left\{\psi \in H: K^{\prime} \cup\{\phi\} \nvdash \psi\right\}=\{\psi \in H$ : $E \cup\{\phi\} \nvdash \psi\}$ then $E$ is preferred and optimal.

Proof. We only show the first property, the second being similar. Let $E \subseteq K^{\prime}$ s.t. $E$ is a MinSHS. One can easily see that $E$ is also an optimal solution since we have $H^{\prime}=\{\psi \in H$ : $\left.K^{\prime} \cup\{\phi\} \nvdash \psi\right\}=H^{\prime \prime}=\{\psi \in H: E \cup\{\phi\} \nvdash \psi\}$ and $S^{\prime}=\left\{\psi \in S: K^{\prime} \cup\{\phi\} \vdash \psi\right\}=S^{\prime \prime}=\{\psi \in$ 
$S: E \cup\{\phi\} \vdash \psi\}$. Assume that $E$ is not preferred. Then there exists a MinSHS $E^{\prime} \subseteq K$ s.t. $H^{\prime} \subset\left\{\psi \in H: E^{\prime} \cup\{\phi\} \nvdash \psi\right\}$ and $S^{\prime} \subseteq\left\{\psi \in S: E^{\prime} \cup\{\phi\} \vdash \psi\right\}$, or $H^{\prime} \subseteq\left\{\psi \in H: E^{\prime} \cup\{\phi\} \nvdash \psi\right\}$ and $S^{\prime} \subset\left\{\psi \in S: E^{\prime} \cup\{\phi\} \vdash \psi\right\}$. Using the fact that $f$ and $g$ associate only non zero weights, $W\left(E^{\prime}, I\right)>W\left(K^{\prime}, I\right)$ holds and we obtain a contradiction since $K^{\prime}$ is an optimal solution. As a consequence, $E$ is a preferred MinSHS.

Example 3. We here consider a case of doctor-patient communication. The propositional variables $S_{1}, S_{2}, S_{3}$ and $S_{4}$ refer to four distinct symptoms; and $D_{1}$ and $D_{2}$ refer to two distinct diseases. The doctor knows the following formula:

$$
\phi=\left(\left(S_{1} \wedge S_{2}\right) \rightarrow D_{1}\right) \wedge\left(S_{3} \rightarrow D_{2}\right)
$$

The patient wants to share the symptoms $S=\left\{S_{1}, S_{2}, S_{3}\right\}$ among the four symptoms that he has, but he also wants to hide that he has the diseases $H=\left\{D_{1}, D_{2}\right\}$. In addition, we define the function $f$ and $g$ on respectively $H$ and $S$ as follows:

- $f\left(D_{1}\right)=2$ and $f\left(D_{2}\right)=3$;

- $g\left(S_{1}\right)=3, g\left(S_{2}\right)=2$, and $g\left(S_{3}\right)=4$.

Thus, we consider the following weighted SHI-frame: $\left(\left(\left\{S_{1}, S_{2}, S_{3}, S_{4}\right\}, \phi, H, S\right), f, g\right)$. The optimal solutions are sol ${ }_{1}=\left\{S_{1}, S_{2}, S_{3}\right\}$, sol $_{2}=\left\{S_{1}, S_{3}\right\}$, sol $l_{3}=\left\{S_{1}, S_{2}, S_{3}, S_{4}\right\}$, sol $4=$ $\left\{S_{1}, S_{3}, S_{4}\right\}$, which have all the weight 9 . Furthermore, sol ${ }_{1}$ and sol 2 are preferred MinSHS, while sol $_{3}$ and sol $_{4}$ are preferred MaxSHS.

In the optimal solutions, no difference is made between the shared and hidden pieces of knowledge. In order to avoid this fact, we consider in the following definition the notion of Pareto optimality (PO).

Definition 15 (PO-Solution). Given a weighted SHI-frame $I=(F, f, g)$ with $F=(K, \phi, H, S)$, a subset $K^{\prime} \subseteq K$ is said to be a PO-solution if, for all $K^{\prime \prime} \subseteq K$, the following properties are satisfied:

- if $\sum_{\psi \in H^{\prime \prime}} f(\psi)>\sum_{\psi \in H^{\prime}} f(\psi)$ then $\sum_{\psi \in S^{\prime \prime}} g(\psi)<\sum_{\psi \in S^{\prime}} g(\psi)$; and

- if $\sum_{\psi \in S^{\prime \prime}} f(\psi)>\sum_{\psi \in S^{\prime}} f(\psi)$ then $\sum_{\psi \in H^{\prime \prime}} g(\psi)<\sum_{\psi \in H^{\prime}} g(\psi)$.

where $H^{\prime}=\left\{\psi \in H: K^{\prime} \cup\{\phi\} \nvdash \psi\right\}, H^{\prime \prime}=\left\{\psi \in H: K^{\prime \prime} \cup\{\phi\} \nvdash \psi\right\}, S^{\prime}=\left\{\psi \in S: K^{\prime} \cup\{\phi\} \vdash\right.$ $\psi\}$, and $S^{\prime \prime}=\left\{\psi \in S: K^{\prime \prime} \cup\{\phi\} \vdash \psi\right\}$.

We use $W H\left(K^{\prime}, I\right)$ and $W S\left(K^{\prime}, I\right)$ to denote respectively $\sum_{\psi \in H^{\prime}} f(\psi)$ and $\sum_{\psi \in S^{\prime}} g(\psi)$.

The following proposition can be shown in the same way as Proposition 4.

Proposition 5. Given a weighted SHI-frame $I=(F, f, g)$ with $F=(K, \phi, H, S)$ and $K^{\prime} \subseteq K$, if $K^{\prime}$ is a PO-solution of $I$, then we have the two following properties:

- for all $E \subseteq K^{\prime}$, if $E$ is a MinSHS s.t. $\left\{\psi \in S: K^{\prime} \cup\{\phi\} \vdash \psi\right\}=\{\psi \in S: E \cup\{\phi\} \vdash \psi\}$ then $E$ is preferred and a PO-solution;

- for all $E \subseteq K$ with $K^{\prime} \subseteq E$, if $E$ is a MaxSHS s.t. $\left\{\psi \in H: K^{\prime} \cup\{\phi\} \nvdash \psi\right\}=\{\psi \in H$ : $E \cup\{\phi\} \nvdash \psi\}$ then $E$ is preferred and a PO-solution. 


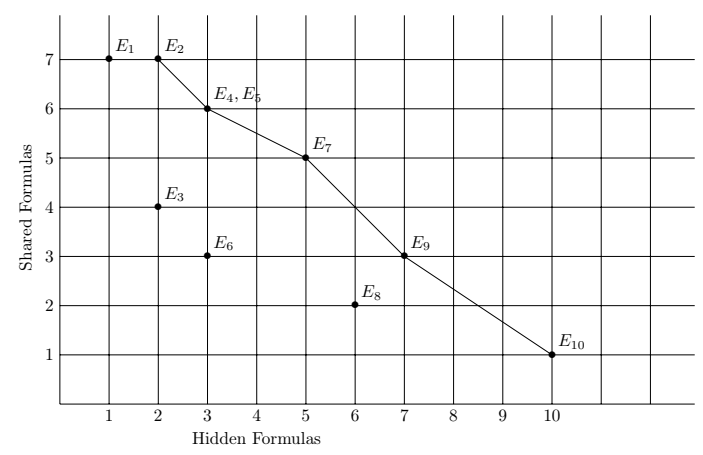

Figure 4: An illustration about PO-solutions

For instance, in Figure 4, we show all the preferred MinSHSes of a given weighted SHIframe $(F, f, g)$ with $F=(K, \phi, H, S)$. The $x$-axis and $y$-axis represent respectively the weights associated with the hidden formulas $(f)$ and those associated with the shared formulas $(g)$. Let us first note that a point can represent more than one preferred MinSHS, as is the case of the point corresponding to $E_{4}$ and $E_{5}$. Clearly, the set $E_{2}$ is a PO-solution, while $E_{1}$ is not because we have:

- $\sum_{\psi \in H_{1}} f(\psi)<\sum_{\psi \in H_{2}} f(\psi)$, and

- $\sum_{\psi \in S_{1}} g(\psi)=\sum_{\psi \in S_{2}} g(\psi)$,

where $H_{1}=\left\{\psi \in H: E_{1} \cup\{\phi\} \nvdash \psi\right\}, H_{2}=\left\{\psi \in H: K_{2} \cup\{\phi\} \nvdash \psi\right\}, S_{1}=\left\{\psi \in S: K_{1} \cup\{\phi\} \vdash\right.$ $\psi\}$, and $S_{2}=\left\{\psi \in S: E_{2} \cup\{\phi\} \vdash \psi\right\}$. In fact, the set of the PO-solutions occurring in Figure 4 is $\left\{E_{2}, E_{4}, E_{5}, E_{7}, E_{9}, E_{10}\right\}$, which is known in multiobjective optimization as Pareto frontier (e.g. see [9]).

Example 4. Consider the weighted SHI-frame $I=((K, \phi, H, S), f, g)$ such that:

- $K=$ \{age, gender, single, OS, clickstream, location, playing\}

- $H=\{2$ : single, 5 : price-discrimination, 3 : personal-information, 6 : political-views, 5 : employability

- $S=\{2$ : single, $3:$ age $, 2:$ gender, $5:$ location, $3:$ playing, $3:$ preferences $\}$

- $\phi=\bigwedge\{$

OS $\rightarrow$ price-discrimination, clickstream $\rightarrow$ personal-information, playing $\rightarrow$ employability, age $\wedge$ gender $\wedge$ location $\rightarrow$ political-views, \} clickstream $\rightarrow$ preferences

where each expression $w$ : e in $H$ (resp. S) means that $f(e)=w($ resp. $g(e)=w)$.

In the previous weighted SHI-frame, we consider that the principal agent is a buyer and the secondary agent is a seller. For instance, the implication OS $\rightarrow$ price-discrimination refers to the fact that if the seller knows the buyer's OS then he can apply a strategy of price 
discrimination to the buyer based on this information, and the application of such a strategy is not desired by the buyer, which is modeled by the fact that the formula (a propositional variable) price-discrimination belongs to the set $H$ in the considered weighted SHI-frame. Let us note that the formula single belongs to both sets $H$ and $S$ with the same weight. Intuitively, this can be seen as a manner to represent the fact that the possible gain in sharing this piece of knowledge is the same as hiding it. As a consequence, sharing the formula single or not depends on the other shared pieces of knowledge. Furthermore, using the fact that the secondary agent has the implication playing $\rightarrow$ employability and the facts that employability belongs to $H$ with the weight 5 and playing belongs to $S$ with the weight 3 , we know that there is no PO-solution that contains playing. As a side note, playing $\rightarrow$ employability refers to the fact that playing video games is used to determine the employability of a person for given works.

One of the interesting PO-solutions is $E=\{$ age, location, single, clickstream $\} . W e$ have:

- $W H(E, F)=f($ price-discrimination $)+f($ employability $)+$ $f$ (political-views $)=16$, and

- $W S(E, F)=g($ single $)+g($ age $)+g($ location $)+g($ preferences $)=13$.

In addition, we have $W H(E \backslash\{$ single $\})=18$ and $W S(E \backslash\{$ single $\})=11$, which particularly means $W(E, F)=W(E \backslash\{$ single $\}, F)$. Furthermore, we have $W H(E \cup$ \{gender $\}, I)=12$ and $W H(E \cup\{$ gender $\}, I)=15$. In this case, even if $W(E \cup\{$ gendre $\}, I)<W(E, I)$, the set $E \cup\{$ gender $\}$ is also a PO-solution. Indeed, by reducing the weights associated with knowledge hiding, gender increases the weight associated with knowledge sharing.

\section{Conclusion and Perspectives}

In this paper, we introduced a logic-based framework for reasoning about knowledge access, which is based on the use of the inference process in classical propositional logic. We consider in this framework that each agent has access to every piece of knowledge that can be derived from the pieces knowledge in her possession using this process. That being said, our approach can be directly adapted to other processes of inference, in particular those of non-classical logics. We proposed a concept called minimal hiding subset (MHS) for dealing with situations where an agent has to hide certain pieces of knowledge, and we showed that the computation of the MHSes can be carried out through the computation of maximal consistent subsets. In addition, we introduce a natural counterpart of the concept of MHS called minimal sharing subset (MSS) that, for its part, can be used for dealing with situations where an agent has to share particular pieces of knowledge. Similarly to the computation of MHSes, we showed that the computation of the MSSes can be achieved by using the computation of minimal inconsistent subsets. Then, combinations of the previous two concepts were proposed in order to capture different types of compromises between knowledge sharing and hiding. Further, we introduced a simple weightbased approach, which is based on associating integers with the pieces of knowledge that an agent wants to hide and also those that she wants to share. In this context, we described the optimal solutions in Pareto sense.

In our future work, we intend to provide a further analysis of the proposed logic-based framework and potential real-world applications. We also plan to propose methods for generating different structures described in this paper, in particular MHSes and MSSes. Moreover, future work includes taking into account dynamic contexts by mainly allowing the principal agent to update her purpose as well as weights. The use of other processes of inference instead of the classical one is as well an important perspective. 


\section{References}

[1] Carlos E. Alchourrón, Peter Gärdenfors, and David Makinson. On the Logic of Theory Change: Partial Meet Contraction and Revision Functions. Journal of Symbolic Logic, 50(2):510-530, 1985.

[2] Meriem Ammoura, Yakoub Salhi, Brahim Oukacha, and Badran Raddaoui. On an MCS-based inconsistency measure. International Journal Approximate Reasoning, 80:443-459, 2017.

[3] Franz Baader, Ian Horrocks, Carsten Lutz, and Ulrike Sattler. An Introduction to Description Logic. Cambridge University Press, 2017.

[4] Salem Benferhat, Didier Dubois, and Henri Prade. Some Syntactic Approaches to the Handling of Inconsistent Knowledge Bases: A Comparative Study Part 1: The Flat Case. Studia Logica, 58(1):17-45, 1997.

[5] Philippe Besnard and Anthony Hunter. Elements of Argumentation. MIT Press, 2008.

[6] Patrick Blackburn, Maarten de Rijke, and Yde Venema. Modal Logic, volume 53 of Cambridge Tracts in Theoretical Computer Science. Cambridge University Press, 2001.

[7] Zhi-Zhong Chen and Seinosuke Toda. The Complexity of Selecting Maximal Solutions. Information and Comput., 119(2):231-239, 1995.

[8] J. E. Doran, S. Franklin, Nicholas R. Jennings, and Timothy J. Norman. On Cooperation in Multi-Agent Systems. The Knowledge Engineering Review, 12(3):309-314, 1997.

[9] Matthias Ehrgott. Multicriteria Optimization (2. ed.). Springer, 2005.

[10] Maria Fasli. Agent Technology For E-Commerce. John Wiley \& Sons, Inc., USA, 2007.

[11] Shaheen Fatima, Sarit Kraus, and Michael J. Wooldridge. Principles of Automated Negotiation. Cambridge University Press, 2014.

[12] Éric Grégoire, Yacine Izza, and Jean-Marie Lagniez. Boosting MCSes Enumeration. In Proceedings of the Twenty-Seventh International Joint Conference on Artificial Intelligence, IJCAI 2018, 2018, Stockholm, Sweden., pages 1309-1315, 2018.

[13] Anthony Hunter and Sébastien Konieczny. On the Measure of Conflicts: Shapley Inconsistency Values. Artificial Intelligence, 174(14):1007-1026, 2010.

[14] Sébastien Konieczny and Ramón Pino Pérez. Logic Based Merging. Journal of Philosophical Logic, 40(2):239-270, 2011.

[15] Sarit Kraus. Automated Negotiation and Decision Making in Multiagent Environments. In 3rd European Agent Systems Summer School, EASSS 2001, Prague, Czech Republic, Selected Tutorial Papers, volume 2086 of Lecture Notes in Computer Science, pages 150-172. Springer, 2001.

[16] Mark H. Liffiton, Alessandro Previti, Ammar Malik, and João Marques-Silva. Fast, Flexible MUS Enumeration. Constraints, 21(2):223-250, 2016.

[17] Liviu Panait and Sean Luke. Cooperative Multi-Agent Learning: The State of the Art. Autonomous Agents and Multi-Agent Systems, 11(3):387-434, 2005.

[18] Christos H. Papadimitriou and David Wolfe. The Complexity of Facets Resolved. Journal of Computer and System Sciences, 37(1):2-13, 1988.

[19] Christos H. Papadimitriou and Mihalis Yannakakis. The Complexity of Facets (and Some Facets of Complexity). Journal of Computer and System Sciences, 28(2):244-259, 1984.

[20] Iyad Rahwan, Sarvapali D. Ramchurn, Nicholas R. Jennings, Peter Mcburney, Simon Parsons, and Liz Sonenberg. Argumentation-based Negotiation. The Knowledge Engineering Review, 18(4):343375, 2003.

[21] Stuart Russell and Peter Norvig. Artificial Intelligence: A Modern Approach, chapter 7. Prentice Hall Press, Upper Saddle River, NJ, USA, 3rd edition, 2009.

[22] Larry J. Stockmeyer. The polynomial-time hierarchy. Theoretical Computer Science, 3(1):1 - 22, 1976.

[23] Jose M. Such, Agustín Espinosa, and Ana García-Fornes. A Survey of Privacy in Multi-Agent Systems. The Knowledge Engineering Review, 29(3):314-344, 2014. 